\title{
Localized nonlinear waves on quantized superfluid vortex filaments in the presence of mutual friction and a driving normal fluid flow
}

\author{
Rehan Shah and Robert A. Van Gorder* \\ Mathematical Institute, University of Oxford, Andrew Wiles Building, Radcliffe Observatory Quarter, Woodstock Road, \\ Oxford, OX2 6GG, United Kingdom
}

(Received 20 September 2015; revised manuscript received 17 January 2016; published 22 March 2016)

\begin{abstract}
We demonstrate the existence of localized structures along quantized vortex filaments in superfluid helium under the quantum form of the local induction approximation (LIA), which includes mutual friction and normal fluid effects. For small magnitude normal fluid velocities, the dynamics are dissipative under mutual friction. On the other hand, when normal fluid velocities are sufficiently large, we observe parametric amplification of the localized disturbances along quantized vortex filaments, akin to the Donnelly-Glaberson instability for regular Kelvin waves. As the waves amplify they will eventually cause breakdown of the LIA assumption (and perhaps the vortex filament itself), and we derive a characteristic time for which this breakdown occurs under our model. More complicated localized waves are shown to occur, and we study these asymptotically and through numerical simulations. Such solutions still exhibit parametric amplification for large enough normal fluid velocities, although this amplification may be less uniform than would be seen for more regular filaments such as those corresponding to helical curves. We find that large rotational velocities or large wave speeds of nonlinear waves along the filaments will result in more regular and stable structures, while small rotational velocities and wave speeds will permit far less regular dynamics.
\end{abstract}

DOI: 10.1103/PhysRevE.93.032218

\section{INTRODUCTION}

Quantum turbulence is an active area of current research and spans several related areas as part of the theory of superfluidity including vortex generation, interaction, and reconnection of closely spaced vortex lines and phase transition problems influenced by quantum vortices [1]. Characterized by highly disordered sets of one-dimensional singularities and defects, the theory of superfluid turbulence is also perceived to be a variation of string field theory as it is used in the study of chaotic motion of objects possessing nonlinear and nonlocal interactions and reconnections. The stochastic dynamics of vortex filaments, particularly in quantum fluids, possesses significant potential to aid in the understanding of superfluid turbulence, a term coined by Feynman in 1955 [2]. This describes the appearance of a disordered set of quantised vortex lines termed vortex tangles in superfluids such as $\mathrm{He}$ II for velocity values above a critical value [3]. What is interesting to note is that equations such as the Biot-Savart Law describing the nonlocal dynamics of vortex-line motion provide valuable information about the properties of vortex tangles, which in turn form the essence of quantum turbulence. The complexity of this problem originates from the coupling of the deterministic motion of the vortex filaments and random collisions of the vortex loops, which motivates the reduction of the Biot-Savart model to a simpler local induction approximation (LIA). In the classical limit (where mutual friction effects are ignored), the LIA can be mapped into a cubic nonlinear Schrödinger (NLS) equation.

Unlike in a classical fluid, the motion of a thin vortex filament in a superfluid such as ${ }^{4} \mathrm{He}$ is modeled taking into account mutual friction, such as that discussed in the HVBK model [4-6]. A number of fundamental results were provided

*Robert.VanGorder@maths.ox.ac.uk by Vinen [7-10]. Mutual friction also plays a role in the dynamics of vortex filaments in ${ }^{3} \mathrm{He}[11,12]$. The self-induced motion of a single quantized vortex filament is governed by the Biot-Savart integral. Nonlocal dynamics under the BiotSavart integral are often approximated by the local induction approximation (LIA), provided that such an approximation is reasonable for the specific physical scenario being described. At finite temperatures, a quantized vortex is also influenced by mutual friction. Replacing the nonlocal term with the LIA and accounting for mutual friction in the normal and binormal directions, Schwarz [13] obtained a kind of quantum LIA (qLIA), which accounts for mutual friction and the interaction with a normal fluid. This model is given in nondimensional form by

$\mathbf{v}=\kappa \mathbf{t} \times \mathbf{n}+\alpha \mathbf{t} \times(\mathbf{U}-\kappa \mathbf{t} \times \mathbf{n})-\alpha^{\prime} \mathbf{t} \times(\mathbf{t} \times(\mathbf{U}-\kappa \mathbf{t} \times \mathbf{n}))$.

Here $\mathbf{U}$ is the dimensionless normal fluid velocity, $\mathbf{v}=\mathbf{r}_{t}$ is the velocity of the filament, $\mathbf{t}$ and $\mathbf{n}$ are the unit tangent and unit normal vectors to the vortex filament, $\kappa$ is the curvature, and $\alpha$ and $\alpha^{\prime}$ are dimensionless mutual friction coefficients which are small (except near the $\lambda$ point; for reference, the $\lambda$ point is the temperature $(\approx 2.17 \mathrm{~K}$, at atmospheric pressure) below which normal fluid helium transitions to superfluid helium [14]). In the limit $\left(\alpha, \alpha^{\prime}\right) \rightarrow(0,0)$, we recover the classical Da Rios equations for the motion of a vortex filament in a classical fluid [15-17].

The small deformations of vortex lines are referred to as Kelvin waves [18], which serve as the primary mechanism to account for the low-temperature decay of superfluid turbulence in the absence of dissipation through mutual friction. The appearance of large vortex rings in chaotic quantized vortices in He II aids in shedding further light on phase transition problems. The key question "To what extent is the dynamics of a set of vortex line able to reproduce the properties of real hydrodynamic turbulence?" [1] can thus be answered by 
studying the behavior of vortex filaments not in classical fluids, but in quantum fluids.

A number of studies exist on the solutions to the quantum LIA given in (1). In the $\alpha, \alpha^{\prime} \rightarrow 0$ limit, these solutions should collapse into solutions of the classical LIA. One highly important class of solutions to the classical LIA would be the one-soliton solution found by Hasimoto [19], by way of what is now referred to as the Hasimoto transformation, which puts the classical LIA into correspondence with the cubic NLS. While a number of solutions to the quantum LIA have been studied either numerically or analytically, the Hasimoto one-soliton has never been extended to the quantum LIA. Recently, the Hasimoto transformation was extended to the quantum LIA (1) in the case where $\alpha|\mathbf{U}|=O\left(\alpha^{2}\right)$ [i.e., $|\mathbf{U}|=O(\alpha)$ was sufficiently small so as to be neglected] in [20]. Defining a complex scalar function

$$
\psi(s, t)=\kappa(s, t) \exp \left(i \int_{0}^{s} \tau(\hat{s}, t) d \hat{s}\right),
$$

where $\kappa(s, t)$ is the local curvature and $\tau(s, t)$ is the local torsion of the filament, respectively, it was shown that the evolution equation for $\psi(s, t)$ takes the form

$$
\begin{aligned}
\dot{\psi}= & i A(t) \psi+\left(i\left(1-\alpha^{\prime}\right)+\alpha\right) \psi_{s s}+\left(\frac{\left(1-\alpha^{\prime}\right) i}{2}-\alpha\right) \\
& \times\left|\psi^{2}\right| \psi-\frac{\alpha}{2} \psi \int_{0}^{s}\left\{\psi(\hat{s}, t) \psi_{s}(\hat{s}, t)^{*}-\psi(\hat{s}, t)^{*} \psi_{s}(\hat{s}, t)\right\} d \hat{s} .
\end{aligned}
$$

Equation (3), obtained from the quantum Hasimoto formulation, is a complex coefficient Ginsburg-Landau equation (GLE) with an extra dissipation term, which for classical fluids (when $\alpha, \alpha^{\prime}=0$ ) reduces to the cubic nonlinear Schrödinger equation (NLS) [19]. The presence of a standing soliton on a vortex filament has been demonstrated theoretically by Hasimoto [19] for classical fluids and by Van Gorder [20] for quantum fluids under Eq. (3). Experimentally, Hopfinger and Browand [21] observed solitary waves on vortex filaments when studying turbulent flow in a rotating container. Such results are useful in demonstrating the interplay between solutions of integrable models (such as the cubic NLS equation) and vortex filament solutions under the LIA.

Equation (2) allowed for a study of Stokes waves, onesolitons, similarity solutions, and other traveling wave solutions along quantized vortex filaments in superfluid helium under mutual friction effects [20]. Each of these solutions generalizes known results for the classical LIA. This map from the quantum LIA to Eq. (3) is a completely natural generalization of the classical LIA to cubic NLS map pioneered by Hasimoto [19], which in its own right has motivated both experimental and theoretical work over the past 40 years. As one example, Hopfinger and Browand [21] studied turbulent flow in a rotating container, and determined that the theoretical solutions predicted by Hasimoto [19] do actually occur along vortex filaments in practical experiments.

Unlike solitons or regular Kelvin waves (which take the form of pure helical vortex filaments) which maintain a constant shape over time, it is possible to search for highly localized waves along quantized vortex filaments in superfluid helium. Recently, a special class of such solutions were shown to exist in the very cold limit where $\alpha, \alpha^{\prime} \rightarrow 0$, corresponding to the classical limit. In particular, breathers along quantized vortex filaments [22,23] have been obtained for the classical LIA valid for very cold temperatures (near temperatures of $0 \mathrm{~K}$ ). However, as it is known that the mutual friction effects and the coupling to the normal fluid flow play large roles in the structural dynamics of a vortex filament for the warmer superfluid limit (temperatures above about $1.0 \mathrm{~K}$ ), it is worthwhile to search for highly localized vortex filament structures in the presence of these quantum effects. Obtaining a framework for studying these highly localized waves along quantized vortex filaments, and then determining the qualitative influence of mutual friction and normal fluid velocities on the behavior of said waves, is the goal of this work.

In Sec. II, we outline the theoretical model which will allow us to study localized waves along quantized vortex filaments in superfluid helium. We obtain a new complex scalar equation, since the existing quantum Hasimoto transformation is of limited use in the regime where normal fluid effects dominate. We are able to discuss general properties of the solutions to this equation, and then relate them back to qualitative features of the quantized vortex filaments. Once a solution is known, one may obtain the vortex filament in either Cartesian coordinates or in terms of curvature and torsion. We elect to do the former, since it permits direct visualization of the vortex filament curve. In Sec. III we obtain some asymptotic results for various vortex filament structures under our formulation. Then, in Sec. IV, a number of numerical solutions are provided to demonstrate the variety of structures possible under the formulation.

In the small normal fluid velocity regime, dissipative mutual friction effects dominate, as was true in the case for solitary waves in the very low-temperature regime. However, when the normal fluid velocity is large enough, we observe a parametric amplification of the localized perturbations along the filaments, which was not possible to see in classical formulations such as [22,23], which neglected mutual friction and normal fluid coupling, nor in the low-temperature limit for the quantum case considered in [20], where normal fluid contributions were taken to be negligibly small [assuming $|\mathbf{U}|=O(\alpha)$ ]. This amplification is akin to the DonnellyGlaberson instability [24-26] observed in regular Kelvin waves along quantized vortex filaments. In Sec. V we give a discussion of the results, as well as routes for future work.

\section{COMPLEX SCALAR PDE FROM THE QUANTUM LIA}

To account for the case of non-negligible normal fluid velocity when $\mathbf{U}$ is not necessarily small in magnitude, we refrain from employing the quantum Hasimoto transformation (3). Instead, we will derive a new formulation for a different complex scalar function, proceeding like in [27]. The function will not have the simple interpretation where the amplitude is the curvature of the filament, and the phase is an integral of the torsion. However, the formulation will permit large magnitude normal fluid velocity effects, which one expects will have a significant influence on the highly localized waves along quantized vortex filaments. 
Differentiating with respect to the arclength variable $s$, and performing several vector manipulations, we have that the quantum LIA (1) becomes

$$
\begin{aligned}
\dot{\mathbf{t}}= & \frac{\partial}{\partial s}\left\{\left(1-\alpha^{\prime}|\mathbf{t}|^{2}\right) \mathbf{t} \times \mathbf{t}_{s}-\alpha\left[\left(\mathbf{t} \cdot \mathbf{t}_{s}\right) \mathbf{t}-|\mathbf{t}|^{2} \mathbf{t}_{s}\right]\right\} \\
& +\frac{\partial}{\partial s}\left\{\alpha \mathbf{t} \times \mathbf{U}-\alpha^{\prime} \mathbf{t} \times(\mathbf{t} \times \mathbf{U})\right\} .
\end{aligned}
$$

Here we have made use of standard vector calculus identities, and we have also taken $\mathbf{t}$ to be a unit vector. Taking $\mathbf{t}$ to be a unit vector, as is standard, Eq. (4) simplifies slightly to the equation

$$
\dot{\mathbf{t}}=\frac{\partial}{\partial s}\left\{\left(1-\alpha^{\prime}\right) \mathbf{t} \times \mathbf{t}_{s}+\alpha \mathbf{t}_{s}+\alpha \mathbf{t} \times \mathbf{U}-\alpha^{\prime}(\mathbf{t} \cdot \mathbf{U}) \mathbf{t}\right\} .
$$

By introducing a complex-valued function $v(s, t), v: \mathbb{R}^{2} \mapsto$ $\mathbb{C}$, the unit tangential vector $\mathbf{t}$ can be written as

$$
\mathbf{t}=\left(\frac{v+v^{*}}{2}, \frac{v-v^{*}}{2 i}, \sqrt{1-|v|^{2}}\right) .
$$

It is worth noting that both $\frac{v+v^{*}}{2}=\operatorname{Re}(v)$ and $\frac{v-v^{*}}{2 i}=\operatorname{Im}(v)$ are real valued and hence $\mathbf{t}$, as written, is a vector with real components. Moreover, it is simple to show that $|\mathbf{t}|=1$; hence $\mathbf{t}$ is always a unit vector. Note that $|v| \leqslant 1$ by assumption. Placing (6) into (5), we obtain a system

$$
\begin{aligned}
\frac{\partial}{\partial t}\left(\frac{v+v^{*}}{2}\right) & =\operatorname{rhs}_{1}\left[v, v^{*}\right], \quad \frac{\partial}{\partial t}\left(\frac{v-v^{*}}{2 i}\right)=\operatorname{rhs}_{2}\left[v, v^{*}\right], \\
\frac{\partial}{\partial t}\left(\sqrt{1-\left|v^{2}\right|}\right) & =\operatorname{rhs}_{3}\left[v, v^{*}\right],
\end{aligned}
$$

where the $\operatorname{rhs}_{k}\left[v, v^{*}\right]$ is a complicated function of $v, v^{*}$ and their derivatives in $s$. (These expressions were obtained via Maple and checked by hand. The explicit forms are omitted for brevity.) Since the first two equations are related through complex conjugations, adding the first equation to $i$ times the second gives

$$
\begin{aligned}
\dot{v} & =\frac{\partial}{\partial t}\left(\frac{v+v^{*}}{2}\right)+i \frac{\partial}{\partial t}\left(\frac{v-v^{*}}{2 i}\right) \\
& =\operatorname{rhs}_{1}\left[v, v^{*}\right]+i \operatorname{rhs}_{2}\left[v, v^{*}\right] .
\end{aligned}
$$

Substituting the form of the right-hand side, we obtain

$$
\begin{aligned}
\dot{v}= & \frac{\partial}{\partial s}\left\{i\left(1-\alpha^{\prime}\right) \frac{v_{s}}{\sqrt{1-|v|^{2}}}+\alpha v_{s}+\frac{i\left(1-\alpha^{\prime}\right)\left(v^{2} v_{s}^{*}-|v|^{2} v_{s}\right)}{2 \sqrt{1-|v|^{2}}}+\frac{\alpha^{\prime} U_{3}|v|^{2} v}{\sqrt{1-|v|^{2}}}\right. \\
& \left.-\frac{\alpha^{\prime} U_{3} v}{\sqrt{1-|v|^{2}}}-i \alpha U_{3} v-\left(U_{1}+i U_{2}\right)\left[\frac{\alpha^{\prime}}{2}|v|^{2}-\frac{i \alpha}{\sqrt{1-|v|^{2}}}\right]+\frac{\alpha^{\prime}}{2}\left(i U_{2}-U_{1}\right) v^{2}\right\},
\end{aligned}
$$

where $\mathbf{U}=\left(U_{1}, U_{2}, U_{3}\right)$. A remark worth making is that the condition $\frac{\partial}{\partial t}\left(\sqrt{1-|v|^{2}}\right)=\operatorname{rhs}_{3}\left[v, v^{*}\right]$ was not used in obtaining this equation. This can be treated as a consistency condition and should be satisfied by any unit vector solution $\mathbf{t}$. For instance, the condition holds if we align the filament along the $z$ coordinate.

It is then assumed that the solution is oriented with the tangent vector $\mathbf{t}$, so that the coordinate frame is $(x, y, z)$ for simplicity. It is also assumed that the filament is aligned along the $z$ axis. As shown by Shivamoggi in [28], the normal fluid flow along the $x$ or $y$ axis will not have much qualitative influence on the shape of the filament. Therefore, we can set the components of the normal fluid velocity orthogonal to the orientation of the filament, $U_{1}$ and $U_{2}$, to zero, while maintaining the qualitative properties of the filament solutions. Furthermore, observe that

$$
v_{s}+\frac{v^{2} v_{s}^{*}-|v|^{2} v_{s}}{2}=\left(1-|v|^{2}\right)^{3 / 2} \frac{\partial}{\partial s}\left(\frac{v}{\sqrt{1-|v|^{2}}}\right) .
$$

After these simplifications, Eq. (9) becomes

$$
\begin{aligned}
\dot{v}= & \frac{\partial}{\partial s}\left[i\left(1-\alpha^{\prime}\right)\left(1-|v|^{2}\right) \frac{\partial}{\partial s}\left(\frac{v}{\sqrt{1-|v|^{2}}}\right)\right. \\
& \left.+\alpha v_{s}-U_{3}\left(\alpha^{\prime} \sqrt{1-|v|^{2}}+i \alpha\right) v\right] .
\end{aligned}
$$

While not at all similar to the equation obtained under the Hasimoto transformation, we note that this equation should encode the relevant physics present under that model in the limit where $U_{3} \rightarrow 0$. Furthermore, once a solution to (11) is known, the position vector $\mathbf{r}(s, t)=[x(s, t), y(s, t), z(s, t)]$ can be recovered from the function $v(s, t)$, viz.,

$$
\mathbf{r}(s, t)=\left[\begin{array}{c}
\int_{0}^{s} \operatorname{Re}(v(\sigma, t)) d \sigma+x_{0}(t) \\
\int_{0}^{s} \operatorname{Im}(v(\sigma, t)) d \sigma+y_{0}(t) \\
\int_{0}^{s} \sqrt{1-|v(\sigma, t)|^{2}} d \sigma+z_{0}(t)
\end{array}\right]
$$

It has been shown in the literature [13,28] that $\alpha^{\prime}<\alpha \ll 1$ and that $\alpha^{\prime}$ does not strongly influence the qualitative behavior of solutions. Therefore, the $\alpha^{\prime}$ terms can be dropped to reduce the PDE in Eq. (11) to

$$
\dot{v}=i \frac{\partial}{\partial s}\left\{\left(1-|v|^{2}\right) \frac{\partial}{\partial s}\left(\frac{v}{\sqrt{1-|v|^{2}}}\right)\right\}+\alpha v_{s s}-i \alpha U_{3} v_{s} .
$$

Equation (13) and Eq. (11) give qualitatively similar solutions, and therefore it is sufficient to consider solutions of Eq. (13). From (13) we see that the time evolution for $v$ involves three qualitatively distinct terms. The first term in (13) is the LIA in the classical limit $\alpha \rightarrow 0$; hence under the present formulation, 
the representation of the classical LIA is exactly

$$
\dot{v}=i \frac{\partial}{\partial s}\left\{\left(1-|v|^{2}\right) \frac{\partial}{\partial s}\left(\frac{v}{\sqrt{1-|v|^{2}}}\right)\right\} .
$$

Since both this and the Hasimoto transformation are equivalent to the classical LIA, then for each solution to the classical LIA under the Hasimoto map (i.e., the cubic NLS equation), there must exist a corresponding solution to (14). The second term in Eq. (13), $\alpha v_{s s}$, is a diffusion term, which results in dissipation of the filament in the presence of mutual friction. The third term in (13), $-i \alpha U_{3} v_{s}$, holds the influence of the normal fluid velocity, $|\mathbf{U}|=U_{3}$. This term may amplify waves along the vortex filament, leading to strong changes in the filament structure.

Since $\alpha \ll 1$, a regular perturbation expansion may be attempted of the form $v(s, t)=v_{0}(s, t)+\alpha v_{1}(s, t)+O\left(\alpha^{2}\right)$. This was done under the alternate formulation (3) for solitons and other regular waves in [20]. The $O(1)$ term corresponds to a solution $v_{0}(s, t)$ for a classical vortex filament, while the $O(\alpha)$ equation represents the first-order correction. While the equation giving the first order correction is linear in $v_{1}$, it has complicated functional coefficients in terms of $s$ and $t$, through the dependence on $v_{0}(s, t)$. Such equations can be very difficult to work with, which is why we avoid perturbation on the quantum Hasimoto model in the first place, for the types of highly localized waves we wish to study. Hence an alternative approach must be sought out in order to determine the behavior of solutions to this model of quantized vortex filament dynamics. Before doing this, the mathematical properties of Eq. (13) are studied, to get a better understanding of the general qualitative behavior of solutions. Then, in Sec. III, we shall obtain approximate solutions to this equation corresponding to specific localized structures. In Sec. IV, we obtain numerical solutions for a wider variety of behaviors.

\section{A. Stability of small perturbations in (11)}

This section analyzes the stability of small perturbations along line filaments governed by Eq. (11). Note that a line filament will correspond to $v(s, t)=0$. It is useful to consider small-amplitude perturbations of a line filament in the form of Kelvin waves, so we consider

$$
v(s, t)=\beta e^{i(k s-c(t))+r(t)},
$$

where $|\beta| \ll 1$, while $c(t)$ and $r(t)$ are real functions to be determined. Then, neglecting $O\left(\beta^{2}\right)$ terms, Eq. (11) gives

$$
-i \dot{c}(t)+\dot{r}(t)=\left\{-\left(1-\alpha^{\prime}\right) k^{2}-U_{3} \alpha^{\prime} k\right\} i-\alpha\left(k^{2}-U_{3} k\right),
$$

which implies, since $c(t)$ and $r(t)$ are real, that $c(t)=(1-$ $\left.\alpha^{\prime}\right) k^{2} t+U_{3} \alpha^{\prime} k t$ and $r(t)=-\alpha k\left(k-U_{3}\right) t$. If $U_{3}<k$, which means the normal fluid velocity is less than the wave number of the perturbation, then $r(t)<0$ for $\alpha>0, k>0$ and the perturbations are stable. On the other hand, if $U_{3}>k$, then the perturbations grow and the solution is unstable.

Therefore, in the small $U_{3}$ case, it is expected that solutions to the PDE will be stable, while for large $U_{3}$, they will be unstable. This is essentially the statement of the DonnellyGlaberson instability [24-26] for the model (11). Under the quantum LIA, results like this were shown for regular helical vortex filaments in [29], while recently such results were shown under the quantum form of the nonlocal Biot-Savart dynamics in [30]. What this means is that, for small $U_{3}$, there should be no drastic changes in the solutions between the quantum Hasimoto map and the PDE (11), since both are essentially the dissipative limit. On the other hand, when $U_{3}$ is large, the PDE in Eq. (11) should be considered as the reliable model, since solutions to the quantum Hasimoto map are no longer representative due to the fact that the model (3) was derived under the assumption that $\alpha|\mathbf{U}|=O\left(\alpha^{2}\right)$. Another point to be noted is that $\alpha^{\prime}$ does not qualitatively influence the structure of these perturbations, which adds further support to our choice to neglect $\alpha^{\prime}$, and to use Eq. (13) in place of Eq. (11).

\section{B. Influence of the dissipative term}

In this section, the influence of the $\alpha v_{s s}$ term in Eq. (13) is considered. It is worthwhile to consider a PDE given in conservation form as an initial value problem

$$
w_{t}=i \frac{\partial}{\partial s}(J[w]), \quad w(s, 0)=W(s), \quad s \in \mathbb{R}, \quad t>0 .
$$

Here $J$ is a nonlinear differential operator in the spatial variable, $s$, with the property $(J[w])^{*}=J\left[w^{*}\right]$. It is useful to note that the complex conjugate $w^{*}$ can be written as $w_{t}^{*}=-i \frac{\partial}{\partial s}\left(J\left[w^{*}\right]\right)$. Multiplying Eq. (17) by $w^{*}$ and the complex conjugate of (17) by $w$ and then adding gives

$$
\frac{\partial}{\partial t}|w|^{2}=i\left(w^{*} \frac{\partial}{\partial s}(J[w])-w \frac{\partial}{\partial s}\left(J\left[w^{*}\right]\right)\right) .
$$

The total wave action (or energy, in some literature) can be defined by

$$
E(t)=\int_{-\infty}^{\infty}|w(s, t)|^{2} d s .
$$

Then, from Eq. (18), assuming that $E(t)$ is sufficiently bounded, it can be found that

$$
\begin{aligned}
\frac{d E}{d t} & =i \int_{-\infty}^{\infty}\left\{w^{*} \frac{\partial}{\partial s}(J[w])-w \frac{\partial}{\partial s}\left(J\left[w^{*}\right]\right)\right\} d s \\
& =i \int_{-\infty}^{\infty}\left\{J\left[w^{*}\right] w_{s}-J[w] w_{s}^{*}\right\} d s,
\end{aligned}
$$

where the last line follows from an integration by parts and also from the assumption that $|w| \rightarrow 0$ as $|s| \rightarrow \infty$ and $J$ is finite such that $|J[w]|<\infty$ as $|w| \rightarrow 0$, all sufficiently rapidly.

Equation (20) gives the change in the wave action over time. Having dealt with the change in energy for the system (17), it is then worth considering a related system with a diffusion term added, that is

$\hat{w}_{t}=i \frac{\partial}{\partial s}(J[\hat{w}])+\alpha \hat{w}_{s s}, \quad \hat{w}(s, 0)=W(s), \quad s \in \mathbb{R}, \quad t>0$.

A remark to make is that the system (21) has the same initial condition as (17). Let $\hat{E}(t)$ denote the quantity (19) with $\hat{w}$ replacing $w$. Then, provided $\hat{E}(t)$ exists, and using the same 
technique as before,

$$
\begin{aligned}
\frac{d \hat{E}}{d t}= & i \int_{-\infty}^{\infty}\left\{J\left[\hat{w}^{*}\right] \hat{w}_{s}-J[\hat{w}] \hat{w}_{s}^{*}\right\} d s \\
& +\alpha \int_{-\infty}^{\infty}\left\{\hat{w}^{*} \hat{w}_{s s}+\hat{w} \hat{w}_{s s}^{*}\right\} d s .
\end{aligned}
$$

It can be noted that

$$
\begin{aligned}
\int_{-\infty}^{\infty}\left\{\hat{w}^{*} \hat{w}_{s s}+\hat{w} \hat{w}_{s s}^{*}\right\} d s & =-\int_{-\infty}^{\infty}\left(\hat{w}_{s}^{*} \hat{w}_{s}+\hat{w}_{s} \hat{w}_{s}^{*}\right) d s \\
& =-2 \int_{-\infty}^{\infty}\left|\hat{w}_{s}\right|^{2} d s
\end{aligned}
$$

Therefore, this results in

$$
\frac{d \hat{E}}{d t}=i \int_{-\infty}^{\infty}\left\{J\left[\hat{w}^{*}\right] \hat{w}_{s}-J[\hat{w}] \hat{w}_{s}^{*}\right\} d s-2 \alpha \int_{-\infty}^{\infty}\left|\hat{w}_{s}\right|^{2} d s .
$$

Since both $w(s, 0)=W(s)$ and $\hat{w}(s, 0)=W(s)$, it can be concluded that $w(s, 0)=\hat{w}(s, 0)$, so for small time $t>0$, it makes sense to then write

$$
\frac{d \hat{E}}{d t}=\frac{d E}{d t}-2 \alpha \int_{-\infty}^{\infty}\left|w_{s}\right|^{2} d s .
$$

For all time $t, \int_{-\infty}^{\infty}\left|w_{s}\right|^{2} d s \geqslant 0$, with equality to zero holding in the case of a constant solution in space. Then, when $\alpha>0$,

$$
\frac{d \hat{E}}{d t} \leqslant \frac{d E}{d t},
$$

for small time. Hence it has been shown that the inclusion of the term $\alpha w_{s s}$ in a general PDE of the form in Eq. (21) results in faster initial decay of the wave action of the solution.

For the PDE in Eq. (13), it can be noted that

$$
J[v]=\left(1-|v|^{2}\right) \frac{\partial}{\partial s}\left(\frac{v}{\sqrt{1-|v|^{2}}}\right)-i \alpha U_{3} v .
$$

Therefore, the same analysis applies to the PDE (13) with initial condition $v(s, 0)$. Thus this means that for the PDE (13), the $\alpha v_{s s}$ term results in more rapid dissipation of the energy of an initial state $v(s, 0)$. Therefore, for very small $t$, it is expected that there will be little change in the initial solution profile $v(s, 0)$; as $t$ increases, more rapid dissipation will be observed. For the vortex filament, there will be more rapid dissipation of an initial disturbance or wave corresponding to $\alpha>0$ rather than in the classical case when $\alpha=0$.

This is consistent with what has been observed in the literature for Kelvin waves along quantum vortex filaments
[29,30] (corresponding to helical filaments). For those cases, the dissipation manifests as a factor which scales as $\mathrm{e}^{-\alpha k_{\text {eff }}^{2} t}$, where $k_{\text {eff }}$ is an effective wave number $\left[k_{\text {eff }}=O(1)\right.$ in $\left.\alpha\right]$ for the helical filament.

\section{ASYMPTOTIC SOLUTIONS}

Before moving on to numerical solutions, we shall first consider some particular regimes within which one may obtain asymptotic solutions for the vortex filament structure.

Due to the complicated form of the PDE (13), in order to obtain asymptotic results we shall assume solutions with modulus that depend on time alone. These solutions can be seen as generalized helix type solutions which gradually amplify or deamplify depending on time. We shall later use numerical simulations to describe solutions with modulus that depends strongly on spatial location.

\section{A. Helix with nonuniform rotation in time}

Let us assume a solution

$$
v(s, t)=A(t) \exp (i \phi(s, t)),
$$

where both $A(t)$ and $\phi(s, t)$ are real-valued functions. This assumption puts (13) into the form of two real equations, given by

$$
\begin{gathered}
\dot{A}=-\sqrt{1-A^{2}} A \phi_{s s}+\alpha A\left(U_{3} \phi_{s}-\phi_{s}^{2}\right), \\
\phi_{t}=-\sqrt{1-A^{2}} \phi_{s}^{2}+\alpha \phi_{s s} .
\end{gathered}
$$

Let us first consider the case where $\phi(s, t)=k s-\theta(t)$, where $k>0$ is a wave number. For nonlinear $\theta(t)$, this describes a helix with nonuniform rotation in time. From Eqs. (29) and (30) we have

$$
\begin{gathered}
\dot{A}=\alpha k\left(U_{3}-k\right) A, \\
\dot{\theta}=\sqrt{1-A^{2}} k^{2},
\end{gathered}
$$

which gives

$$
A(t)=A_{0} \exp \left(\alpha k\left(U_{3}-k\right) t\right)
$$

and

$$
\theta(t)=k^{2} \int_{0}^{t} \sqrt{1-A_{0}^{2} \exp \left(2 \alpha k\left(U_{3}-k\right) \sigma\right)} d \sigma+\theta_{0},
$$

where $0<A_{0}<1$ and $0 \leqslant \theta<2 \pi$ are constant parameters. This gives the solution

$$
v(s, t)=A_{0} \exp \left(\alpha k\left(U_{3}-k\right) t\right) \exp \left(i\left\{k s-k^{2} \int_{0}^{t} \sqrt{1-A_{0}^{2} \exp \left(2 \alpha k\left(U_{3}-k\right) \sigma\right)} d \sigma+\theta_{0}\right\}\right) .
$$

Recovering the filament solution via (12), we find

$$
\mathbf{r}(s, t)=\left[\begin{array}{c}
\frac{A_{0}}{k} \exp \left(\alpha k\left(U_{3}-k\right) t\right) \sin \left(k s-k^{2} \int_{0}^{t} \sqrt{1-A_{0}^{2} \exp \left(2 \alpha k\left(U_{3}-k\right) \sigma\right)} d \sigma+\theta_{0}\right)+\hat{x}_{0}(t) \\
-\frac{A_{0}}{k} \exp \left(\alpha k\left(U_{3}-k\right) t\right) \cos \left(k s-k^{2} \int_{0}^{t} \sqrt{1-A_{0}^{2} \exp \left(2 \alpha k\left(U_{3}-k\right) \sigma\right)} d \sigma+\theta_{0}\right)+\hat{y}_{0}(t) \\
\sqrt{1-A_{0}^{2} \exp \left(2 \alpha k\left(U_{3}-k\right) t\right) s}+z_{0}(t)
\end{array}\right] .
$$


The time evolution of this filament will depend on the relative size of $k$ and $U_{3}$. If $U_{3}<k$, then the solution will gradually decay in amplitude over time, tending to a line filament as $t \rightarrow \infty$. If $U_{3}=k$, then the filament will maintain it's radius of deflection from the central axis for all time. In this case, the rotational motion will become uniform in time. Finally, if $U_{3}>k$, corresponding to the Donnelly-Glaberson instability, then the filament will amplify over time. However, this cannot continue indefinitely, as we assumed $|v(s, t)|<1$ in deriving the model. Therefore, there exists some critical value of time at which the framework will break down as the radius of deflection becomes too large. Physically, this may correspond to breakdown of the vortex filament. In particular, when $U_{3}>k$, we find that the filament will amplify for time $t$ satisfying

$$
0<t<t^{*}=\frac{1}{\alpha k\left(U_{3}-k\right)} \ln \left(\frac{1}{A_{0}}\right),
$$

while breakdown of the model (and perhaps the filament itself) will occur for $t=t^{*}$, after which point the filament no longer exists. For very small $\alpha$, this breakdown time is greatly increased. So, for the ultracold limit, the filament will amplify more slowly, with breakdown taking longer. As $\alpha \rightarrow 0$, there is no longer parametric amplification or decay, and any solutions are eternal (as is true for the classical LIA). As $k \rightarrow U_{3}$, the solution also becomes eternal as the dissipation and amplifying effects are balanced, and we obtain a helical filament curve with constant radius. This recovers qualitative results of [29], which were obtained by more complicated derivations employing the full vector form of the LIA.

\section{B. Compression wave or kink along a helical filament}

We shall now turn our attention to a type of localized nonlinear wave which travels along a helical filament resulting in a compression or expansion of the filament curve. Let us first assume a small amplitude solution, $A(t) \ll 1$, so that the term $1-A^{2} \approx 1$. We also assume now that $A$ can depend on space, but slowly (so, time is the dominant term in $A$ ). First, Eq. (30) gives

$$
\phi_{t}=-\phi_{s}^{2}+\alpha \phi_{s s} .
$$

If we assume a solution of the form

$$
\phi(s, t)=k s-\omega t+\alpha \Psi(S, T),
$$

with fast variables $S=s / \alpha$ and $T=t / \alpha$, and pick $\omega=k^{2}$, we end up scaling $\alpha$ out of the PDE, obtaining

$$
\Psi_{T}+2 k \Psi_{S}+\Psi_{S}^{2}-\Psi_{S S}=0 .
$$

Consider a wave solution $\Psi(S, T)=\eta(S-c T)=\eta(q)$. Then, Eq. (40) is put into the form

$$
(2 k-c) \frac{d \eta}{d q}+\left(\frac{d \eta}{d q}\right)^{2}-\frac{d^{2} \eta}{d q^{2}}=0 .
$$

Note that (41) has the exact solution

$$
\eta(q)=-\ln \{C+\exp ((2 k-c) q)\} .
$$

Returning to original variables, we have obtained the exact solution

$$
\phi(s, t)=k s-k^{2} t-\alpha \ln \left\{C+\exp \left(\frac{2 k-c}{\alpha}(s-c t)\right)\right\} .
$$

In addition to the wave number, the solution has free parameters $c$ and $C$. Again, assuming variations in $A$ are small with $s$, we find that $A(s, t)$ scales like

$$
\begin{aligned}
A(s, t) \sim & A_{0} \exp \left(\frac{C(2 k-c)}{c}\left[C+\exp \left(\frac{2 k-c}{\alpha}(s-c t)\right)\right]^{-1}\right) \\
& +O\left(\alpha^{2}\right)
\end{aligned}
$$

where $0<A_{0} \ll 1$. The $O\left(\alpha^{2}\right)$ term includes the contribution of $U_{3}$, and hence the dynamics arising from interactions with the normal fluid occur at very slow time scales for these particular solutions.

Physically, as $(2 k-c) s \rightarrow-\infty$ (which is the limit where the ln term acts as a constant), we have a helical structure with effective wave number $k$. However, in the limit $(2 k-c) s \rightarrow$ $\infty$ (which is the limit where the ln term grows linearly), we have a helical structure with effective wave number $c-k$. Therefore, the wave $s-c t$ will modify the helix structure as it passes. This can be viewed as a kink or a compression wave, depending on how it acts on the undisturbed helix with wave number $k$. Depending on the parameter values taken, this wave can cause the helix to compress $(c>2 k)$, remain unchanged $(c=2 k)$, unwind $(k<c<2 k)$, annihilate into a line filament $(c=k)$, or reverse in orientation $(c<k)$. Hence this nonlinear wave has a strong influence on the local structure of the vortex filament as it passes. We use (12) to recover the filament curve, and plot representative solutions in Fig. 1. In these plots we fix $\alpha=0.073$, which corresponds to a temperature of $1.5 \mathrm{~K}$ [13]. The value of $C$ is not found to strongly influence the form of the solution, it just should be positive, so we fix $C=1$.

Note that the effect is $O(1)$ in $\alpha$ due to the fast time variable $t / \alpha$. Of course, we still need finite positive $\alpha$ for this formulation to make sense, meaning that we cannot take the limit $\alpha \rightarrow 0$ directly and recover a type of solution to the classical LIA. Indeed, this solution is particular to the quantum LIA.

From the form of $A$, as the wave passes, the amplitude of deflection from the central axis of symmetry is also modified slightly, although it remains positive (no decay to zero) for appropriate parameter values. One could obtain a more accurate approximation or expansion for $A$, but the approximation given is suitable for our purposes.

This solution demonstrates an interesting kind of nonlinear wave which propagates along the vortex filament and changes the geometry of the vortex filament curve as it passes. Such a solution is permitted due to the appearance of two time scales. The first time scale is the standard $O(1)$ time scale with which the macroscopic helical filament rotates. The second time scale permits a small wave to propagate along this filament with time scale $O\left(\alpha^{-1}\right)$, resulting in a possible change in filament geometry as it passes. In the ultracold limit $\alpha \rightarrow 0$, the action of this highly localized wave would be instantaneous, and we would simply recover the static filament geometry exhibiting only rotation in the slower time scale. 


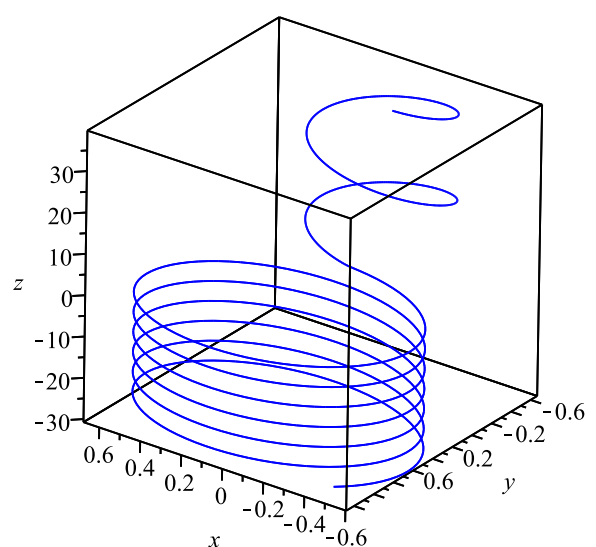

(a)

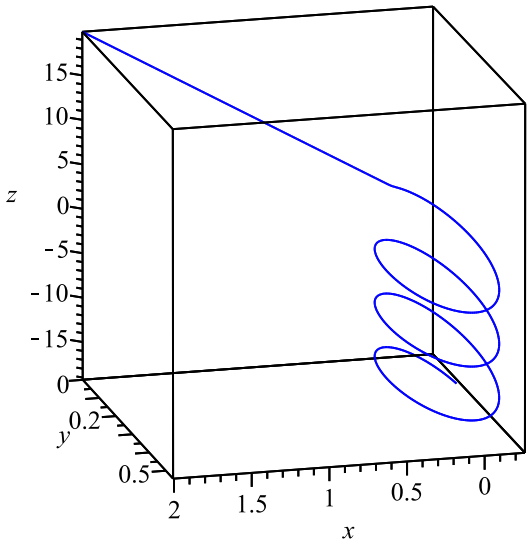

(b)

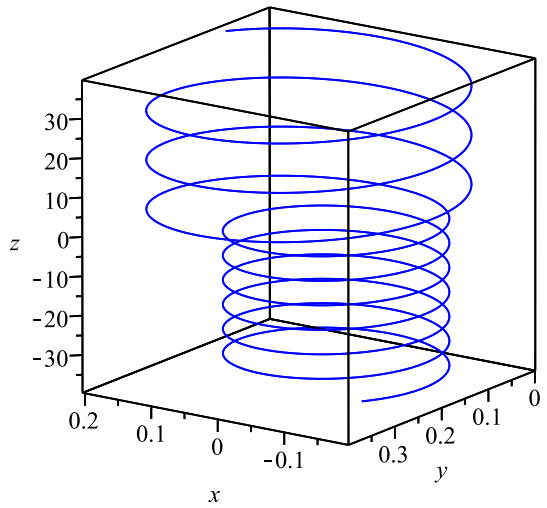

(c)

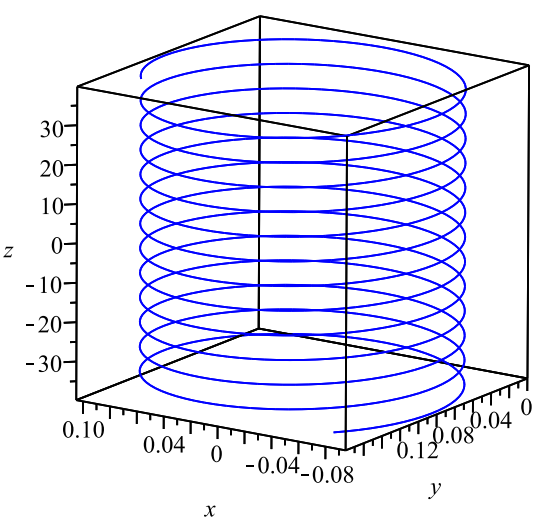

(d)

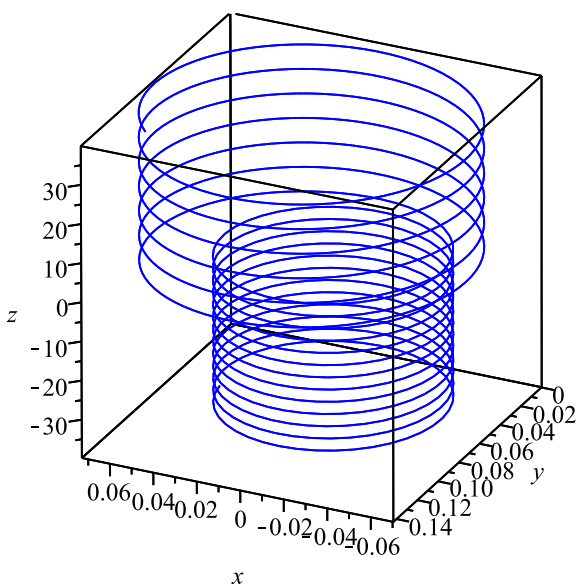

(e)

FIG. 1. Here we plot solutions originating from (43) and (44) for fixed $\alpha=0.073, k=1, C=1$, and $A_{0}=0.1$, given various values of the wave speed parameter $c$ : (a) $c=0.7$, (b) $c=1$, (c) $c=1.5$, (d) $c=2$, and (e) $c=3$. Note that it is sufficient to fix one of $c$ or $k$, since it is their difference which determines the qualitative properties of solutions. These solutions sample the variety of possible behaviors seen when the wave propagating along the vortex filament results in a coiling or decoiling of the helical structure. All plots correspond to $t=0$. As time increases, the helical filaments will rotate, while the interface between the two solution forms will propagate with wave speed $c$ along the filament.

\section{NUMERICAL SIMULATION OF LOCALIZED WAVE SOLUTIONS}

Due to the complicated nature of the governing equation (13), we now turn our attention to numerical simulations. We shall focus on localized wave solutions along rotating filament structures. To this end, consider

$$
v(s, t)=f(\zeta) e^{-i \omega t},
$$

where $\zeta=s-\chi t$ is the wave variable and both of $\omega$ and $\chi$ are nonzero real parameters. This transformation of the solution results in the conversion of (13) into the complex traveling wave ODE

$$
\begin{aligned}
-\chi \frac{d f}{d \zeta}-i \omega f= & i \frac{d}{d \zeta}\left\{\left(1-|f|^{2}\right) \frac{d}{d \zeta}\left(\frac{f}{\sqrt{1-|f|^{2}}}\right)\right\} \\
& +\alpha \frac{d^{2} f}{d \zeta^{2}}-i \alpha U_{3} \frac{d f}{d \zeta}
\end{aligned}
$$

For the sake of numerical calculation, we write $f(\zeta)=$ $f_{R}(\zeta)+i f_{I}(\zeta)$, and we obtain the coupled system of real ODEs

$$
\begin{aligned}
& -\chi \frac{d f_{R}}{d \zeta}+\omega f_{I} \\
& =-\frac{d}{d \zeta}\left\{\left(1-f_{R}^{2}-f_{I}^{2}\right) \frac{d}{d \zeta}\left(\frac{f_{I}}{\sqrt{1-f_{R}^{2}-f_{I}^{2}}}\right)\right\} \\
& \quad+\alpha \frac{d^{2} f_{R}}{d \zeta^{2}}+\alpha U_{3} \frac{d f_{I}}{d \zeta} \\
& -\chi \frac{d f_{I}}{d \zeta}-\omega f_{R} \\
& =\frac{d}{d \zeta}\left\{\left(1-f_{R}^{2}-f_{I}^{2}\right) \frac{d}{d \zeta}\left(\frac{f_{R}}{\sqrt{1-f_{R}^{2}-f_{I}^{2}}}\right)\right\} \\
& \quad+\alpha \frac{d^{2} f_{I}}{d \zeta^{2}}-\alpha U_{3} \frac{d f_{R}}{d \zeta}
\end{aligned}
$$




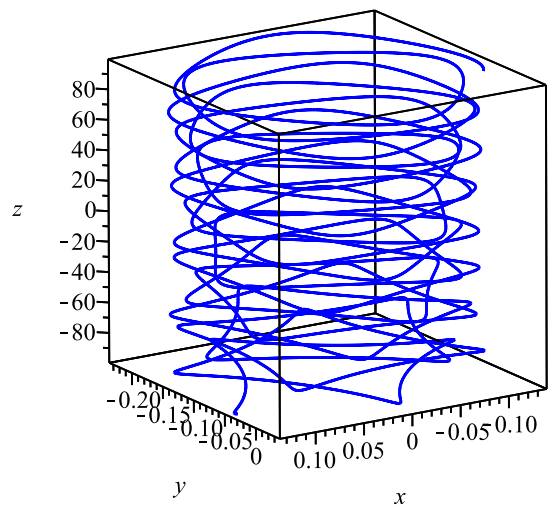

(a)

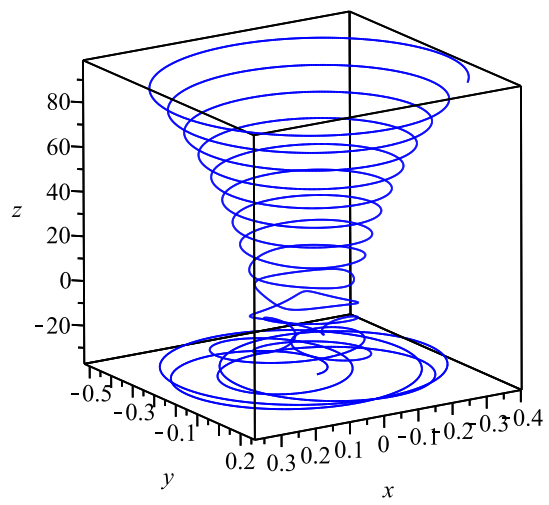

(d)

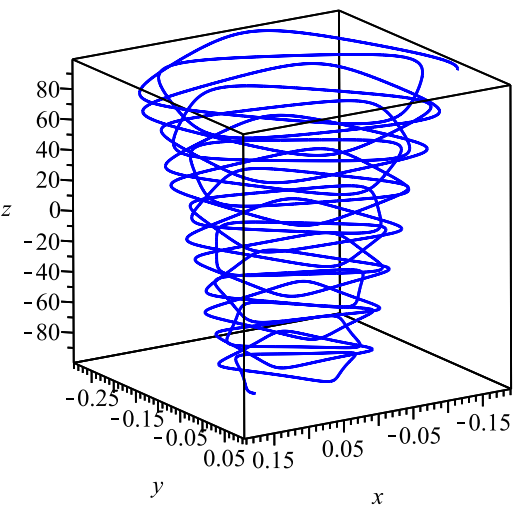

(b)

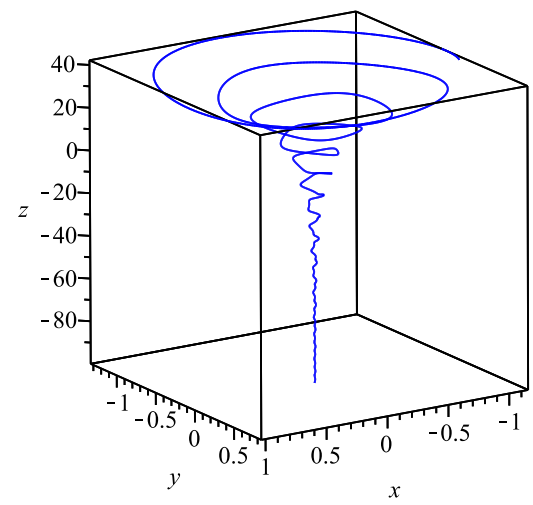

(e)

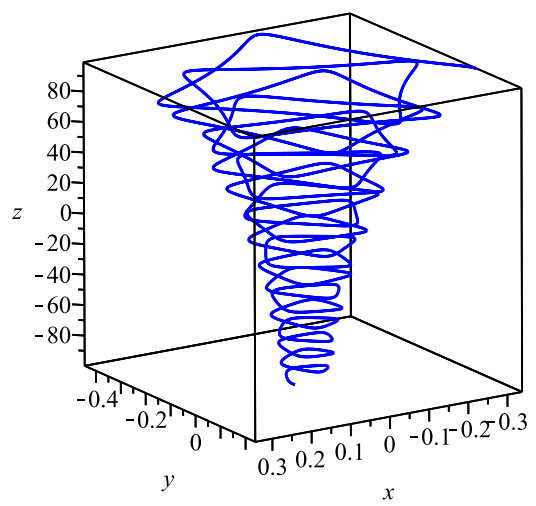

(c)

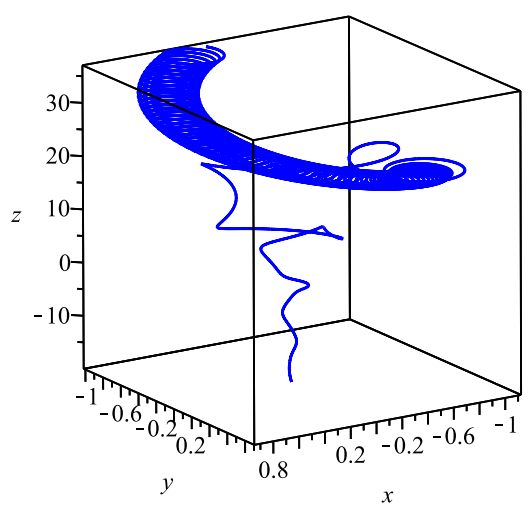

(f)

FIG. 2. Plot of localized structures on vortex filaments for various $\alpha$ and $U_{3}$ : (a) $\alpha=0.006, U_{3}=0$, (b) $\alpha=0.006, U_{3}=2$, (c) $\alpha=$ $0.006, U_{3}=5$, (d) $\alpha=0.073, U_{3}=0$, (e) $\alpha=0.073, U_{3}=2$, and (f) $\alpha=0.073, U_{3}=5$. Here we plot solutions originating from the traveling wave solutions to (47) and (48) for fixed $\chi=1, \omega=1$, and with initial conditions $f_{R}(0)=0.1, f_{I}(0)=0, f_{R}^{\prime}(0)=0$, and $f_{I}^{\prime}(0)=0$. Plots are given at $t=0$ : as these are traveling wave solutions, the filament structures will rotate in time while the structure itself is shifted downward or upward with wave speed $\chi$. Plots are given for $\alpha=0.006$ (corresponding to a temperature of $1.0 \mathrm{~K}$ ) and $\alpha=0.073$ (corresponding to a temperature of $1.5 \mathrm{~K}$ ). We observe amplification as $U_{3}$ increases, and this amplification is more pronounced in the warmer superfluid (larger $\alpha)$ as the coupling between the vortex dynamics and the normal fluid is enhanced as the temperature increases. However, unlike the helical case, here the amplification is not uniform, owing to the more localized structure of solutions. Interestingly, the structures do not destabilize uniformly for large $U_{3}$. Rather, for larger values of $U_{3}$, we actually find some pattern formation. In panel (f), for instance, we obtain a solution which connects a line filament when $s \rightarrow-\infty$ with a twisted helix when $s \rightarrow+\infty$.

Note that one may consider a linear stability analysis around a purely helical solution in order to determine the sign of the real part of the resulting eigenvalues (purely complex eigenvalues will asymptotically give the helix). We find that for the four eigenvalues $\lambda_{j}(j=1,2,3,4)$, when $\chi^{2}+$ $4 \omega>0$,

$$
\min _{j} \operatorname{Re}\left(\lambda_{j}\right)=\frac{U_{3}-\chi}{2} \alpha-\frac{\left|U_{3} \chi-\chi^{2}-2 \omega\right|}{2 \sqrt{\chi^{2}+4 \omega}} \alpha+O\left(\alpha^{2}\right)
$$

and

$$
\max _{j} \operatorname{Re}\left(\lambda_{j}\right)=\frac{U_{3}-\chi}{2} \alpha+\frac{\left|U_{3} \chi-\chi^{2}-2 \omega\right|}{2 \sqrt{\chi^{2}+4 \omega}} \alpha+O\left(\alpha^{2}\right)
$$

Therefore, when $\alpha>0$, there is no localized amplification or decay of the wave if both $\chi=U_{3}$ (the wave speed exactly agrees with the normal fluid velocity) and $\omega=0$ (the rotational part is trivial). Of course, if $\alpha=0$ (the classical limit), then there will be no amplification or decay and the filament structure is eternal. Similar results can be obtained for $\chi^{2}+4 \omega \leqslant 0$. 


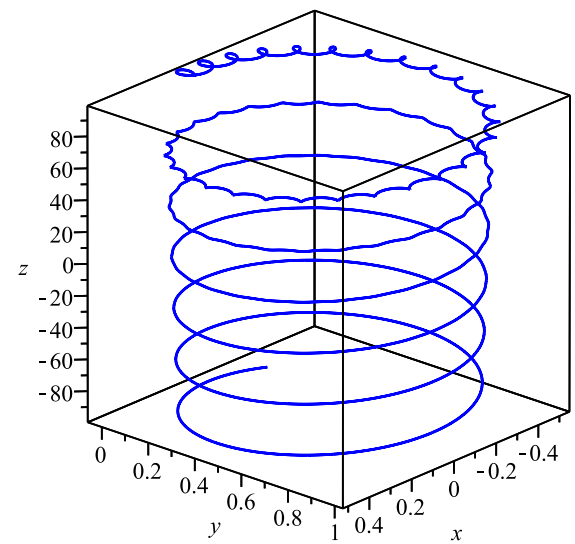

(a)

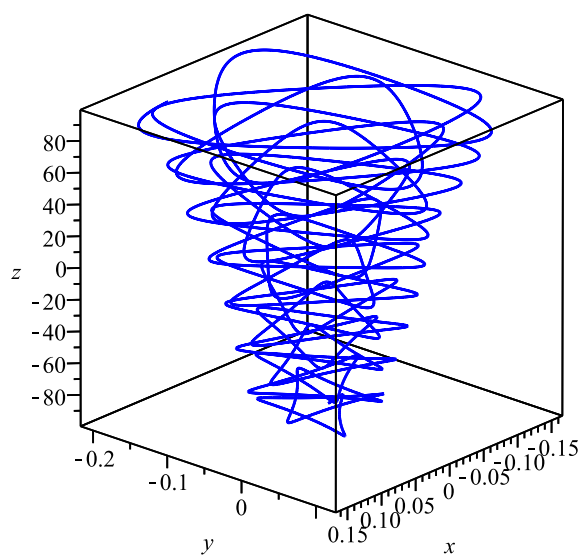

(d)

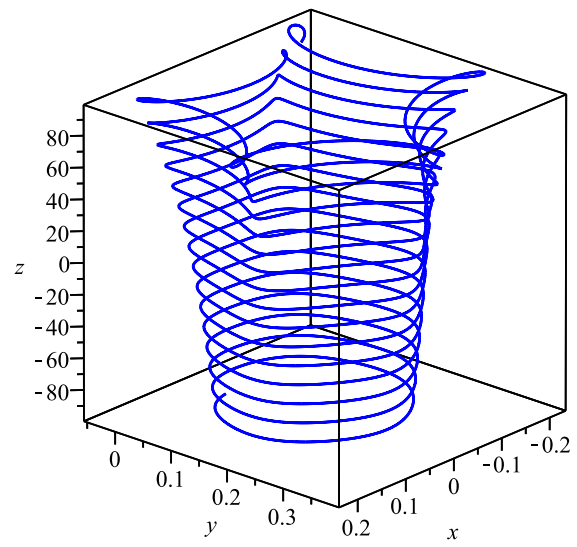

(b)

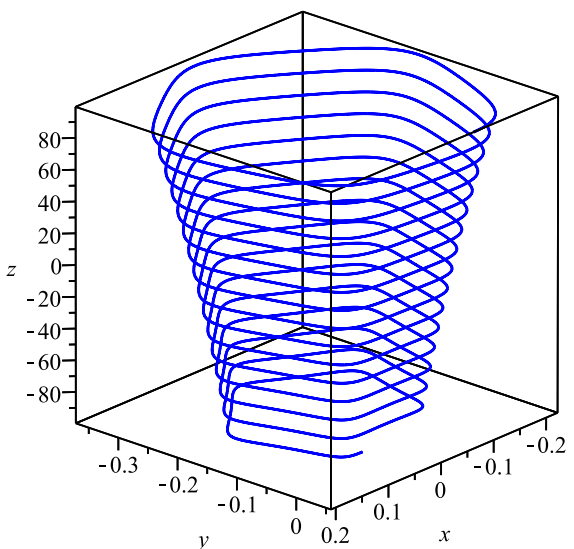

(e)

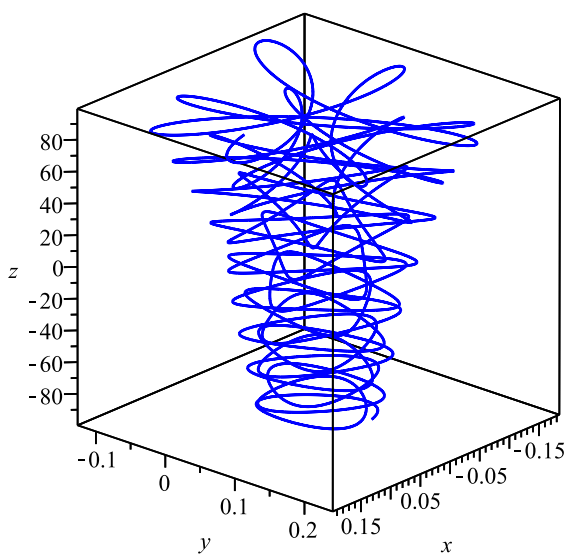

(c)

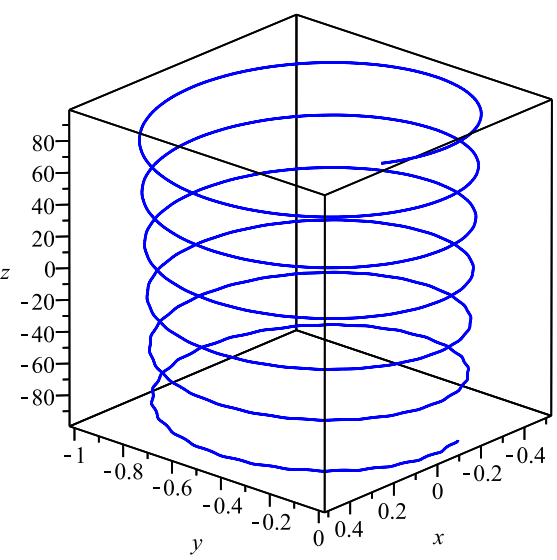

(f)

FIG. 3. Plot of localized structures on vortex filaments for various values of the wave speed $\chi$ : (a) $\chi=-5$, (b) $\chi=-1.5$, (c) $\chi=-0.5$, (d) $\chi=0.5$, (e) $\chi=1.5$, and (f) $\chi=5$. Here we plot solutions originating from the traveling wave solutions to (47) and (48) for fixed $\omega=1$, and with initial conditions $f_{R}(0)=0.1, f_{I}(0)=0, f_{R}^{\prime}(0)=0$, and $f_{I}^{\prime}(0)=0$. Plots are given for $\alpha=0.006$ (corresponding to a temperature of $1.0 \mathrm{~K}$, with similar results holding when $\alpha=0.073$ ) and $U_{3}=2$. Plots are given at $t=0$ : as these are traveling wave solutions, the filament structures will rotate in time while the structure itself is shifted downward or upward with wave speed $\chi$.

For given boundary data and parameter values, the solutions can be obtained numerically. Once this is done, one can use the map (12) to obtain the vortex filament solution, which is given by

$$
\mathbf{r}(s, t)=\left[\begin{array}{c}
\left(\int_{0}^{s} f_{R}(\sigma-\chi t) d \sigma\right) \cos (\omega t)+\left(\int_{0}^{s} f_{I}(\sigma-\chi t) d \sigma\right) \sin (\omega t)+\hat{x}_{0}(t) \\
\left(\int_{0}^{s} f_{I}(\sigma-\chi t) d \sigma\right) \cos (\omega t)-\left(\int_{0}^{s} f_{R}(\sigma-\chi t) d \sigma\right) \sin (\omega t)+\hat{y}_{0}(t) \\
\int_{0}^{s} \sqrt{1-\left(f_{R}(\sigma-\chi t)\right)^{2}-\left(f_{I}(\sigma-\chi t)\right)^{2}} d \sigma+\hat{z}_{0}(t)
\end{array}\right] .
$$

For our numerical simulations, we consider either $\alpha=$ 0.006 or $\alpha=0.073$, which correspond to a temperature of $1.0 \mathrm{~K}$ or $1.5 \mathrm{~K}$, respectively [13]. As temperature decreases, the value of $\alpha$ will decrease, resulting in slower dynamics due to mutual friction and the interaction with the normal fluid. In Fig. 2 we plot solutions for various values of $U_{3}$ to show the amplification effects of increasing the strength of the normal fluid velocity. Note that since we obtain localized structures, rather than uniform global helical structures, the manner of amplification is not always uniform. Note that if we change the sign of $U_{3}$, then the filaments will appear to amplify toward the negative $s$ axis rather than the positive $s$ axis. For larger values of $U_{3}$ we can also observe the emergence of somewhat regular solutions for some parameter values. One such solution, which behaves asymptotically like a line filament as $s \rightarrow-\infty$ and asymptotically like a twisted helix as $s \rightarrow+\infty$, is shown in Fig. 2(f). Note, particularly in Fig. 2(c), the appearance of sharper turns of kinks along what otherwise appears to be a helical structure. These are localized waves that will propagate along the filament curve. These types of waves may only appear over certain regions. In Fig. 2(d), for instance, we see such waves sufficiently close to $s=c t$ at a fixed time $t$, yet away from this $(|s-c t|>10)$ the solution takes on a more helical appearance. 


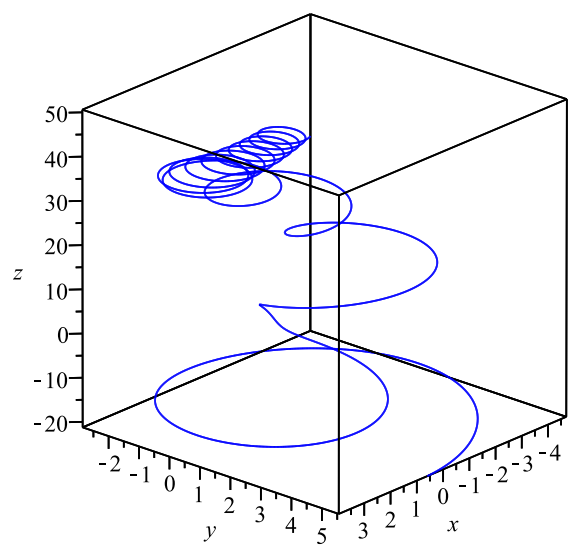

(a)

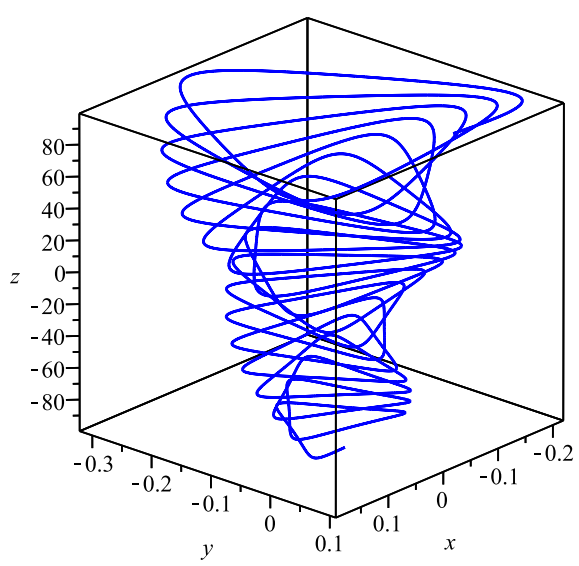

(d)

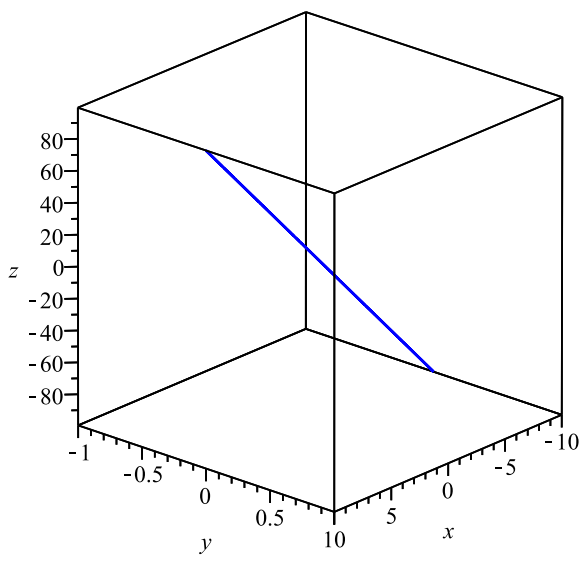

(b)

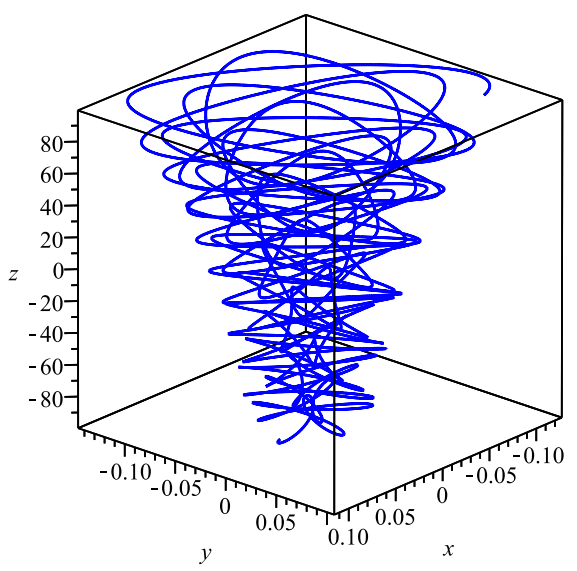

(e)

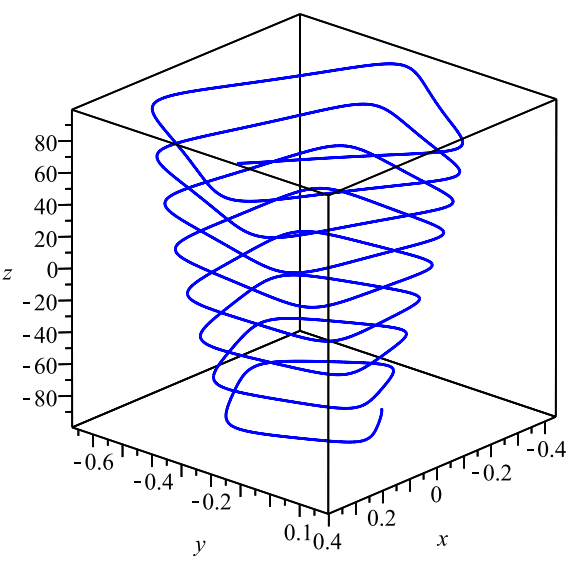

(c)

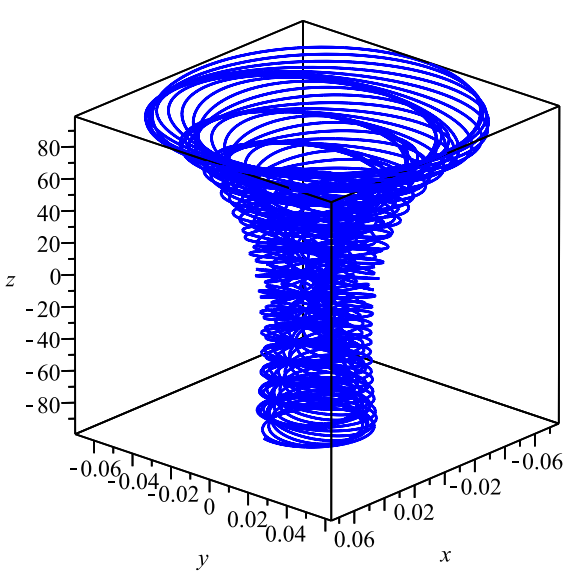

(f)

FIG. 4. Plot of localized structures on vortex filaments for various values of the rotational velocity parameter, $\omega$ : (a) $\omega=-0.1$, (b) $\omega=0$, (c) $\omega=0.2$, (d) $\omega=0.6$, (e) $\omega=2$, and (f) $\omega=15$. Here we plot solutions originating from the traveling wave solutions to (47) and (48) for fixed wave speed $\chi=0.5$ (corresponding to slowly moving waves along the vortex filament), and with initial conditions $f_{R}(0)=0.1$, $f_{I}(0)=0, f_{R}^{\prime}(0)=0$, and $f_{I}^{\prime}(0)=0$. Plots are given for $\alpha=0.006$ (corresponding to a temperature of $1.0 \mathrm{~K}$ ) and $U_{3}=2$. Plots are given at $t=0$ : as these are traveling wave solutions, the filament structures will rotate in time while the structure itself is shifted downward or upward with wave speed $\chi$. Solutions appear to stop existing for large negative $\omega$. If $\omega=0$, the solution will reduce to a line filament. Large $\omega$ corresponds to a rapidly rotating filament structure, and rotational motion appears to dominate the filament structure as we increase $\omega$, smoothing any perturbations of disturbances along the filament curve.

In Fig. 3 we plot the dependence of the solutions on the wave speed parameter, $\chi$. We find that for large wave speeds, that is for high velocity translation of local waves along the vortex filament, there is a regularization of the overall filament structure. This results in a far more uniform filament, even if some small disturbances are present along the filament curve, and appears to suppress the influence of mutual friction and the normal fluid velocity on amplifying or deamplifying the waves as they travel. Therefore, waves with high translational velocity (in either direction along the filament) appear to be more robust against perturbation. On the other hand, slowly moving waves along the filament appear far more susceptible to the effects of various perturbations, and less regular structures emerge. It is therefore in the regime where $|\chi|$ is sufficiently small that we can see vortex filament structures which exhibit great variability in their structure. Note that when $\chi=0$, we would obtain a planar vortex filament, but only in the case where $\alpha=0$. If $\alpha>0$, such a planar solution is not possible, due to induced torsion from the mutual friction terms [31]. Therefore, $\chi=0$ and $\alpha>0$ will result in nonplanar solutions.

Regarding nonlinear waves, note that for large $z$ Fig. 3(b) shows dynamics akin to those corresponding to breathers in [22]. Meanwhile, for negative $z$, the filament takes on the appearance of a helix. Therefore, as the filament amplifies due to the normal fluid component directed along the $z$ axis, planar Kelvin waves which are essentially linear will give way to nonlinear waves with propertied defined by the nonlinearity in the problem. More extreme cases of nonlinear waves are shown in Figs. 3(c) and 3(d). As the wave speed of these waves increases, the nonlinear effects matter less compared the other terms, and the nonlinear waves appear to reduce again to planar Kelvin waves.

In Figs. 4 and 5, we plot the dependence of some of these solutions on the spectral or rotational velocity parameter, 


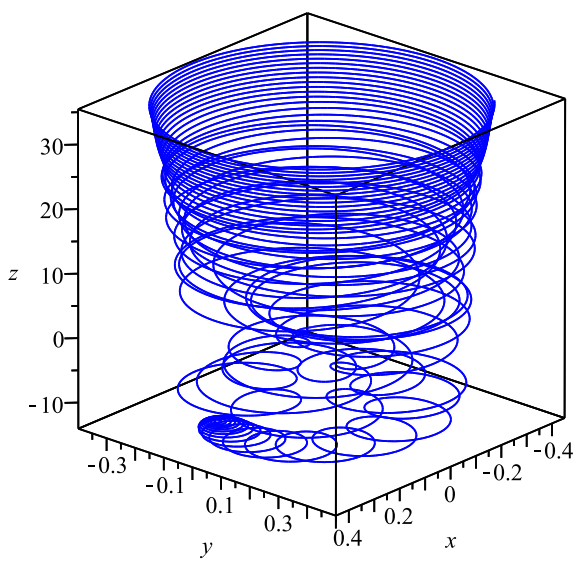

(a)

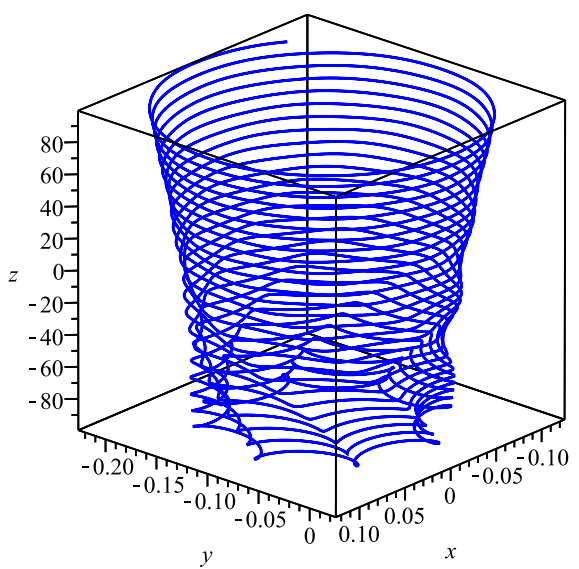

(d)

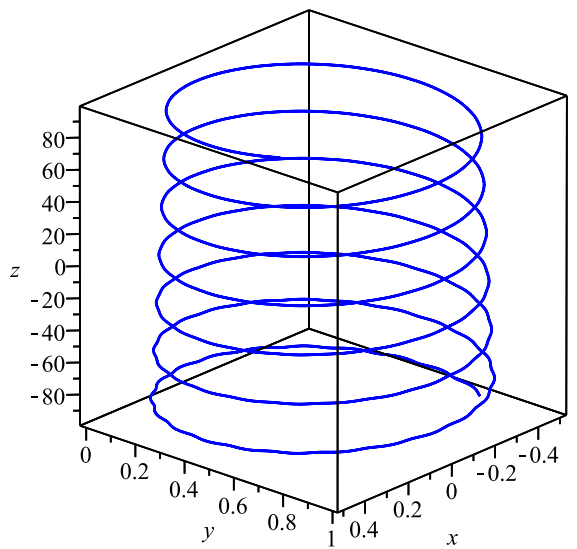

(b)

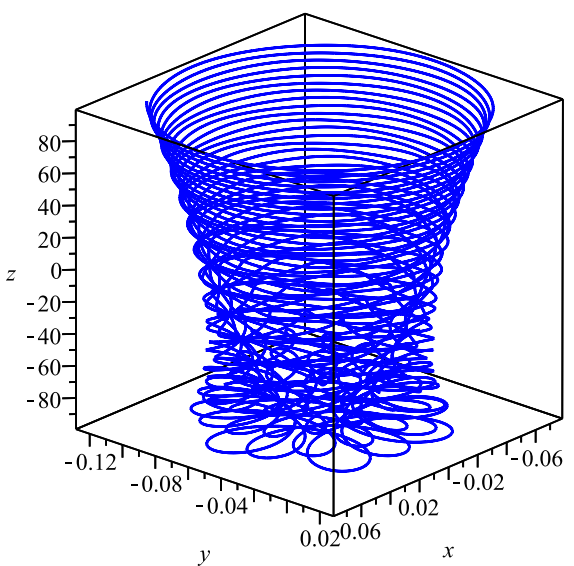

(e)

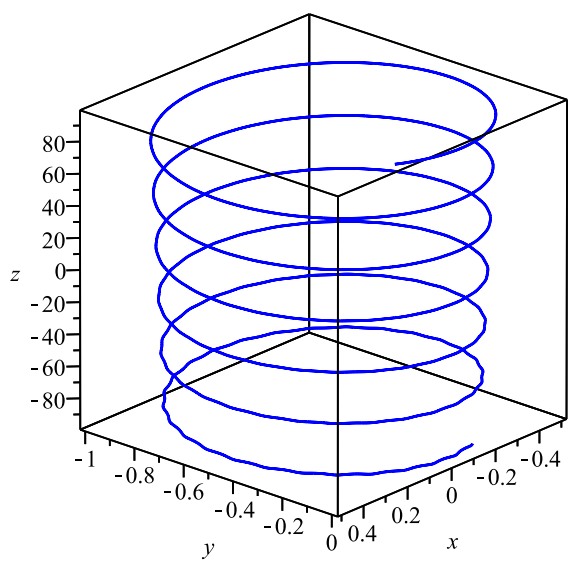

(c)

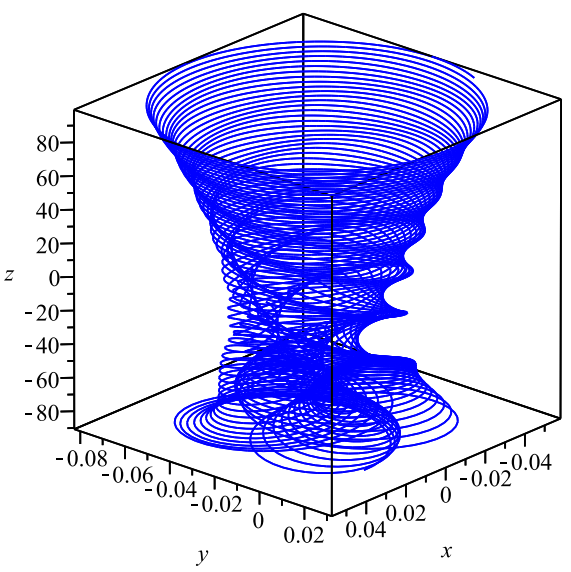

(f)

FIG. 5. Plot of localized structures on vortex filaments for various values of the rotational velocity parameter, $\omega$ : (a) $\omega=-10$, (b) $\omega=-1$, (c) $\omega=1$, (d) $\omega=5$, (e) $\omega=10$, and (f) $\omega=20$. Here we plot solutions originating from the traveling wave solutions to (47) and (48) for fixed wave speed $\chi=5$ (corresponding to rapidly moving waves along the vortex filament), and with initial conditions $f_{R}(0)=0.1, f_{I}(0)=0$, $f_{R}^{\prime}(0)=0$, and $f_{I}^{\prime}(0)=0$. Plots are given for $\alpha=0.006$ (corresponding to a temperature of $1.0 \mathrm{~K}$ ) and $U_{3}=2$. Plots are given at $t=0$ : as these are traveling wave solutions, the filament structures will rotate in time while the structure itself is shifted downward or upward with wave speed $\chi$. Since we have previously shown that rapidly moving waves appear to be more stable, we are able to sample a wider range of possible large magnitude rotational velocities.

$\omega$. First, in Fig. 4, we consider the small wave speed case (taking $\chi=0.5$ ), and demonstrate the dependence of solution structures on the rotational velocity parameter, $\omega$. For small and moderately sized rotational velocity $\omega$, the localized structures along the vortex filament will persist. For large negative $\omega$, the solutions fail to exist. Likely, solutions become unstable for large enough negative $\omega$. The reason for this is that $\omega<0$ corresponds to rotation of the vortex filament against the normal fluid, while $\omega>0$ corresponds to rotation of the vortex filament with the normal fluid. Therefore, when $\omega<0$ and $|\omega|$ is sufficiently large in magnitude, the mathematical solutions do not exist. Physically, these solutions would break down at finite time, and hence are not stable. Meanwhile, for large positive $\omega$, the rotational motion dominates and smooths any perturbations or disturbances along the vortex filament. This results in a regular and tightly coiled structure.

In Fig. 5, we consider the large wave speed case (taking $\chi=5$ ), and again demonstrate the dependence of solution structures on the rotational velocity parameter, $\omega$, for this case. Here solutions are very regular for small magnitude $\omega$, and we find helical filaments. This is in agreement with what was seen in Fig. 3, as when the translational motion of waves along the filament is large, the solutions exhibit a strong degree of regularity. Due to the increase in regularity of the large wave speed solutions, we are able to demonstrate the existence of large negative $\omega$. These solutions exhibit a large degree of disorder near the origin, while for large $|z|$ the solutions tend to appear helical. If we consider large positive $\omega$, then the solutions will appear tightly coiled with patterns forming along the filament curves. For very large positive $\omega$, the solutions will be dominated by rotational motion, akin to what was seen in Fig. 4 for the large positive $\omega$ limit.

Figures 4 and 5 again demonstrate the existence of more localized nonlinear waves along the vortex filaments. These are best observed in Figs. 4(c)-4(e) when the rotational velocity of the filament is sufficiently small. For large rotational velocities, 
there is a smoothing effect, and nonlinear waves can give way to planar Kelvin waves in this regime. See, for instance, Figs. 5(d)-5(f), which demonstrate this regularization or deregularization (depending on the sign of $\chi$ ) of the solutions with time.

Also in the numerical solutions, when $\omega=0$, we always obtain a line filament. When $\chi=0$ and $\alpha=0$ we obtain a planar filament. However, when $\chi=0$ yet $\alpha>0$, we no longer have this planar filament (in agreement with the results of [31] which show that planar filaments are not to be expected under quantum LIA models such as that we consider here due to torsion effects from the coupling with $\alpha$ and $\mathbf{U}$ ).

While a variety of solutions are possible, and the above are only a sampling, these numerical solutions demonstrate that there are indeed rather localized structures possible under our complex scalar PDE formulation of the LIA. Such localized waves along vortex filament still exhibit parametric amplification from the normal fluid coupling (for large enough normal fluid velocity); however, this amplification can be less uniform than what we see for the helical filaments (corresponding to uniform Kelvin waves).

\section{DISCUSSION}

We have obtained a framework for studying quantized vortex filaments in superfluid helium which exhibit strong locality in space and time. In contrast previous results in this area have neglected [22] the effects of mutual friction and normal fluid velocity, although we show that the normal fluid velocity is important, with parametric amplification observed in some regimes. Other previous studies have considered very regular shaped filaments in space, such as solitary waves or helices [20,29]. Highly localized structures along vortex filaments are completely transient, and will not display persistence in time. This may make such solutions ideal for studying rapid changes in vortex filament structure, perhaps leading to turbulence.

In Sec. II we obtained an alternate model for the dynamics of a quantized vortex filament in superfluid helium. This was motivated by the need to include the effects of strong normal fluid velocities directed along the quantized vortex filaments, which were anticipated to have strong qualitative influence over the vortex filament dynamics in the case where strongly localized waves along the filaments was permitted. Models based on classical LIA and the quantum generalization of the Hasimoto transformation neglect such effects. For the highly localized waves along vortex filaments, large structural changes in the filaments resulted when the normal fluid velocity and the temperature were both sufficiently large. Therefore, the consideration of normal fluid effects is essential in the warmer temperature regime if one is to appropriately study temporally localized waves on quantized vortex filaments. On the other hand, when either the magnitude of the normal fluid velocity is small, or the temperature is close enough to the zero-temperature limit (i.e., equal to or below a temperature of about $1.0 \mathrm{~K}$ ), there were minimal structural changes, and one can therefore assume these effects are perturbative. In this latter case, one is justified in studying models such as (3), which is the generalization of the Hasimoto transformation to include mutual friction. Some particular asymptotic scalings are given for this model throughout Sec. II, while some asymptotic solutions (in the case where the modulus of solutions is primarily temporally dependent rather than spatially dependent) are provided in Sec. III. We are able to derive, for helixlike vortex filaments, a characteristic time scale on which solutions can persist in the case of parametric amplification before the reduction to LIA is no longer valid. After this time, the filaments are not guaranteed to exist; indeed, they may collapse, or nonlocal terms from the Biot-Savart dynamics may be needed to properly describe their time evolution. For solutions not exhibiting parametric amplification, the filaments will either maintain their general form or will asymptotically collapse to line filaments.

In Sec. IV, we obtained numerical solutions for various values of $U_{3}$ to show the amplification effects of increasing the strength of the normal fluid velocity. Note that since we obtain localized structures, rather than uniform global helical structures, the manner of amplification is not always uniform, and for certain parameter values, more interesting vortex filament structures were found. When we consider waves moving along the vortex filament, we find that for large wave speeds, there is a regularization of the overall filament structure. Meanwhile, slowly moving waves along the filament appear far more susceptible to the effects of various perturbations, and less regular structures emerge. In this regime, the amplitude of the disturbance will play a much larger role. It is therefore in the small wave speed regime that we can see vortex filaments exhibit great variability in their structure. Likewise, in the limit where rotational velocity of the entire filament is very large, it dominates the filament structure and dynamics in time. When rotational velocity and the wave speed of nonlinear waves along the filament are both small enough, a variety of structures dominated by the nonlinearity inherent in the LIA are possible.

Many of the nonlinear waves observed along filaments occur locally, with the filament behaving like a helical filament asymptotically for $|z| \rightarrow \infty$. Hence the nonlinear wave solutions obtained tend to be local rather than global in nature. This is potentially useful experimentally: one could have boundary conditions consistent with more common helical filaments, yet still hope to observe less regular nonlinear waves along the vortex filaments for appropriate parameter values, as well.

As might be expected from the known Donnelly-Glaberson instability results for Kelvin waves (which correspond to helical vortex filaments $[29,30])$, we observe a parametric amplification of the localized spatiotemporal waves along the quantized vortex filaments. However, while the helical filaments would exhibit a uniform amplification over the entire vortex filament length, the localized waves may exhibit parametric amplification only locally. Therefore, the parametric amplification of such vortex filaments ends up being a local, rather than global, phenomenon. This is perhaps more physically realistic, as it means that the straight segments of a filament are largely unaffected, while sharp turns and waves in the center of the filament are strongly modified, in contrast to a uniform amplification seen in the helical case.

Here we have studied nonlinear waves along a single quantum vortex filament. Such filament configurations constitute one part of the possible dynamics of the vortex lines. Superfluid turbulence involves large deformations of vortex 
loops, collisions and reconnection of vortex lines, the escape of vortex loops from the bulk, and so on [1]. To account for such behaviors, one typically needs the nonlocal formulation of the vortex filament problem, which was recently used to study parametric amplification of Kelvin waves [30] when mutual friction and normal fluid effects were included. Kelvin waves roughly obeying the dynamics expected from the LIA have recently been observed in experiments in superfluid helium [32], lending validity to the view that LIA dynamics are worthy of further study. An interesting area of future work would be the interaction of two or more quantized vortex filaments, each with a highly localized structure such as those reported here. The irregular, transient structures explored in this paper could, perhaps, serve as interesting models for the generation of quantum turbulence, since we expect that their interaction dynamics would be more complicated than the dynamics of interacting regular Kelvin waves (uniformly helical filaments).

On a related note, it may be possible to observe some of the quantized vortex filament structures obtained mathematically. Indeed, the one-soliton was observed experimentally a decade ago [21] after it was predicted theoretically by Hasimoto [19]. The one-soliton is rather idealized, so less regular filament structures obtained due to strong mutual friction and normal fluid velocity effects are certainly possible.
[1] S. K. Nemirovskii, Phys. Rep. 524, 85 (2013).

[2] R. P. Feynman, Prog. Low Temp. Phys. 1, U53 (1955).

[3] S. K. Nemirovskii and W. Fiszdon, Rev. Mod. Phys. 67, 37 (1995).

[4] H. E. Hall and W. F. Vinen, Proc. R. Soc. London A 238, 204 (1956).

[5] H. E. Hall and W. F. Vinen, Proc. R. Soc. London A 238, 215 (1956).

[6] I. L. Bekarevich and I. M. Khalatnikov, Sov. Phys. JETP 13, 643 (1961).

[7] W. F. Vinen, Proc. R. Soc. London A 240, 114 (1957).

[8] W. F. Vinen, Proc. R. Soc. London A 240, 128 (1957).

[9] W. F. Vinen, Proc. R. Soc. London A 242, 493 (1957).

[10] W. F. Vinen, Proc. R. Soc. London A 243, 400 (1958).

[11] N. B. Kopnin and M. M. Salomaa, Phys. Rev. B 44, 9667 (1991).

[12] T. D. C. Bevan, A. J. Manninen, J. B. Cook, A. J. Armstrong, J. R. Hook, and H. E. Hall, Phys. Rev. Lett. 74, 750 (1995).

[13] K. W. Schwarz, Phys. Rev. B 31, 5782 (1985).

[14] L. D. Landau and E. M. Lifshitz, Fluid Mechanics (Addison and Wesley, Redwood City, CA, 1959).

[15] L. S. Da Rios, Rend. Circ. Mat. Palermo 22, 117 (1906).

[16] R. J. Arms and F. R. Hama, Phys. Fluids 8, 553 (1965).
[17] R. L. Ricca, Nature (London) 352, 561 (1991).

[18] Lord Kelvin, Philos. Mag. 5, 155 (1880).

[19] H. Hasimoto, J. Fluid Mech. 51, 477 (1972).

[20] R. A. Van Gorder, Phys. Rev. E 91, 053201 (2015).

[21] E. J. Hopfinger and F. K. Browand, Nature (London) 295, 393 (1982).

[22] H. Salman, Phys. Rev. Lett. 111, 165301 (2013).

[23] M. Umeki, Theor. Comput. Fluid Dyn. 24, 383 (2010).

[24] D. K. Cheng, W. M. Cromar, and R. J. Donnelly, Phys. Rev. Lett. 31, 433 (1973).

[25] W. I. Glaberson, W. W. Johnson, and R. M. Ostermeier, Phys. Rev. Lett. 33, 1197 (1974).

[26] R. M. Ostermeier and W. I. Glaberson, J. Low Temp. Phys. 21, 191 (1975).

[27] R. A. Van Gorder, J. Fluid Mech. 740, 5 (2014).

[28] B. K. Shivamoggi, Eur. Phys. J. B 86, 275 (2013).

[29] R. A. Van Gorder, Phys. Fluids 26, 075101 (2014).

[30] R. A. Van Gorder, Proc. R. Soc. A 471, 20150149 (2015).

[31] R. A. Van Gorder, Proc. R. Soc. A 470, 20140341 (2014).

[32] E. Fonda, D. P. Meichle, N. T. Ouellette, S. Hormoz, and D. P. Lathrop, Proc. Natl. Acad. Sci. USA 111, 4707 (2014). 